\title{
Prevention and management of pain in the neonatal intensive care unit
}

\author{
Sung Shin Kim, MD, PhD \\ Department of Pediatrics, Soonchunhyang University Bucheon Hospital, Soonchunhyang University College of Medicine, Bucheon, Korea
}

Variable degrees of neonatal discomfort, stress, or pain resulting from mild to moderate procedures may occur during routine neonatal intensive care. ${ }^{1)}$ Health care providers are also expected to prevent or treat pain. Preventing and relieving pain in neonates is important because it is a moral obligation and exposure to repeated pain experiences during the neonatal period is known to have short- and long-term adverse effects, including alterations in pain sensitivity and reactivity and the generalized stress arousal system. ${ }^{2)}$ Nociceptive pathways are active and functional in the fetus by 25 weeks of gestation and may provoke a generalized or overstated response to harmful stimuli in newborns. ${ }^{2)}$

Assessing pain in newborns is challenging because of the infants' inability to communicate with the health care providers. ${ }^{3)}$ Therefore, neonatal intensive care units must adopt effective pain assessment tools that consider multiple factors (gestational age and physiological and behavioral responses to pain).

Thus, health care professionals face the dilemma of balancing the need for proper monitoring, testing, and treatment with the need to minimize patient pain and stress. Certain nonpharmacological methods can efficiently decrease pain and discomfort due to routine care measures and minor procedures (e.g., heel stick and phlebotomy) in preterm and term neonates. ${ }^{4}$ These methods include breastfeeding, nonnutritive sucking, swaddling, or facilitated tucking (gently maintaining the arms and legs in a flexed position), skin-to-skin contact (e.g., kangaroo care), and sensorial saturation through massage, touch, voice, and smell.

Pharmacological therapies for neonatal pain control include oral sucrose and other sweet liquids, local analgesia including topical anesthetics and lidocaine, and systematic analgesia including opioid therapy (morphine and fentanyl). ${ }^{5)}$ When sucrose is used, it should be prescribed and tracked as a medication, and the neonate should be monitored for changes in vital signs as well as any clinical signs of aspiration. Whenever pharmacological therapyespecially systematic analgesia-is used, the balance between the benefits and the adverse effects of the intervention must be considered. Opioid therapy, in particular, can have numerous adverse effects including respiratory depression, hypotension, urinary retention, and reduced gut motility.
Kumar et al. $\left.{ }^{6}\right)$ investigated and compared the effectiveness of various nonpharmacological methods of analgesia in newborns in a prospective study conducted at a tertiary care hospital. Three hundred healthy term neonates were divided into 6 groups of 50 newborns each. Groups 1-5, the intervention groups, received one of 5 nonpharmacological interventions (breastfeeding, nonnutritive sucking, rocking, $25 \%$ sucrose, or distilled water), while group 6 , the control group, received no intervention. All patients subsequently received an intramuscular hepatitis B vaccine injection. Interestingly, in contrast to most other studies, Kumar et al. $\left.{ }^{6}\right)$ considered sucrose a nonpharmacological method of intervention, reporting that when breastfeeding is unavailable, other methods such as $25 \%$ sucrose or nonnutritive sucking can be employed in the pain management of newborns.

Preventing pain is an important goal of pain management. Further, it is considerably more difficult to control pain once it has started. A thorough understanding of the pain mechanism is necessary for pain management in newborns. Therefore, every neonatal intensive care unit should adopt an effective pain prevention protocol that includes plans for reducing the number of painful procedures performed as well as a pain assessment and management plan that includes routine pain assessment and pharmacological and nonpharmacological options for minimizing pain.

See the article "Effectiveness and comparison of various nonpharmacological methods of analgesia in newborns" in Volume 63 on page 25 .

\section{Conflicts of interest}

No potential conflict of interest relevant to this article was reported.

\section{Acknowledgments}

This work was supported by the Soonchunhyang University

Corresponding author: Sung Shin Kim, MD, PhD. Department of Pediatrics, Soonchunhyang University Bucheon Hospital, Soonchunhyang University College of Medicine, 170 Jomaru-ro, Bucheon 14584, Korea 
Research Fund.

\section{References}

1. Carbajal R, Rousset A, Danan C, Coquery S, Nolent P, Ducrocq S, et al. Epidemiology and treatment of painful procedures in neonates in intensive care units. JAMA 2008;300:60-70.

2. Committee on Fetus and Newborn and Section on Anesthesiology and Pain Medicine. Prevention and management of procedural pain in the neonate: an update. Pediatrics 2016;137:e20154271.

3. Eriksson M, Campbell-Yeo M. Assessment of pain in newborn infants. Semin Fetal Neonatal Med 2019;24:101003.

4. Carter BS, Brunkhorst J. Neonatal pain management. Semin Perinatol 2017;41:111-6.

5. Bhalla T, Shepherd E, Tobias JD. Neonatal pain management. Saudi J Anaesth 2014;8(Suppl 1):S89-97.

6. Kumar P, Sharma R, Rathour S, Karol S, Karol M. Effectiveness and comparison of various non-pharmacological methods of analgesia in newborns. Clin Exp Pediatr 2020;63:25-9. 